\title{
2 Pharmakokinetische Besonderheiten beim adipösen Patienten
}

\author{
Alexander Kratzer
}

\subsection{Verteilungsvolumen bei Adipositas}

Wie bereits beschrieben, wird das Verteilungsvolumen eines Pharmakons wesentlich durch seine physikochemischen Eigenschaften bestimmt. Insbesondere bei lipophilen Arzneistoffen können beim adipösen Patienten durch den erhöhten Körperfettanteil pharmakokinetisch relevante Unterschiede bezüglich des Verteilungsvolumens auftreten. Während z.B. bei einem normalgewichtigen Mann mittleren Alters der Körperfettanteil im Bereich 18\% bis 24\% liegt, weisen adipöse Patienten häufig Werte von deutlich über 30\% auf. Diese beim adipösen Patienten deutlich vergrößerte Fettmasse führt folglich auch zu einer Vergrößerung der Aufnahmefähigkeit des Körperfett-Kompartiments für lipophile Pharmaka, was schließlich in einem erhöhten Verteilungsvolumen resultiert.

Bei lipophoben Arzneistoffen, die nur in geringem Ausmaß ins Fettgewebe penetrieren, zeigt sich beim adipösen Patienten ebenfalls eine allerdings nur geringfügige Erhöhung des Verteilungsvolumens, da beim Adipösen im Vergleich zum Normalgewichtigen auch die fettfreie Körpermasse (LBW; lean body weight) etwas erhöht ist. Untersuchungen haben gezeigt, dass der Unterschied im Gesamtkörpergewicht (TBW; total body weight) zwischen normalgewichtigen und adipösen Patienten zu ca. 80\% aus der $\mathrm{Zu}$ nahme der Körperfettmasse und zu ca. 20\% aus der Zunahme der fettfreien Masse resultiert (s. Abb. 6).
Hanley et al. (2010) haben diese Erläuterungen in einer Übersichtsarbeit zur Beeinflussung pharmakokinetischer Vorgänge durch Adipositas anschaulich dargelegt (s. Abb. 6).

Die Plasmaproteinbindung, insbesondere die Bindung an Albumin, die ebenfalls wesentlich das Verteilungsvolumen eines Wirkstoffs bestimmt, scheint nach derzeitigem Kenntnisstand durch Adipositas nicht beeinflusst zu werden. Wie bereits in Kapitel VIII.1.2.1 erläutert, ist das Verteilungsvolumen der zentrale pharmakokinetische Parameter zur Abschätzung der Initialdosis. Angesichts obiger Erläuterungen sollte bei Dosierung nach Körpergewicht für lipophile Arzneistoffe mit großem Verteilungsvolumen nach Gesamtkörpergewicht(TBW) dosiert werden, um ausreichende Blutplasmaspiegel zu erzielen.

Bei lipophoben Arzneistoffen ohne nennenswerte Penetration ins Fettgewebe und mit niedrigem Verteilungsvolumen ist es sinnvoll, die Initialdosis nach fettfreier Körpermasse (LBW) zu wählen, da bei Dosierung nach Gesamtkörpergewicht zu hohe Plasmaspiegel resultieren können. Angaben zu Penetration ins Fettgewebe, sowie zum Verteilungsvolumen finden sich häufig bei den Angaben zur Pharmakokinetik in den jeweiligen Fachinformationen. Formeln zur Berechnung der fettfreien Körpermasse finden sich in den Abbildungen 8 und 9 . 


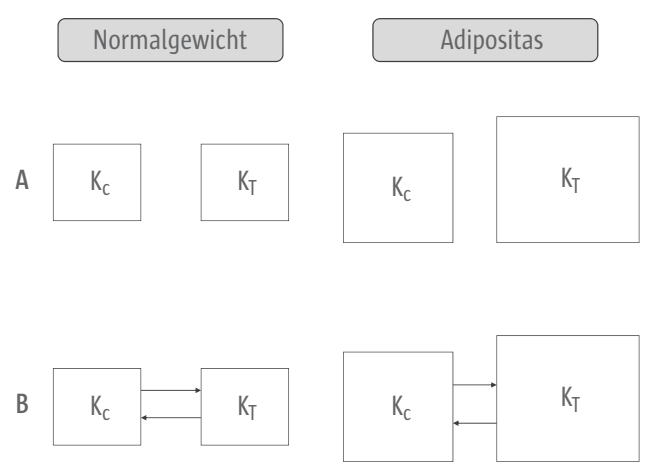

Abb. 6 A: Lipophober Arzneistoff ohne nennenswerte Penetration ins Fettgewebe (Tiefes Kompartiment $\mathrm{K}_{\mathrm{T}}$ ): Das Verteilungsvolumen ist beim adipösen Patienten nur geringfügig erhöht, da auch die fettfreie Körpermasse (Zentrales Kompartiment $K_{c}$ ) nur geringfügig gegenüber normalgewichtigen Patienten erhöht ist. Eine Verteilung ins Fettgewebe findet nicht in nennenswertem Umfang statt. B: Lipophiler Arzneistoff mit Penetration ins Fettgewebe (Tiefes Kompartiment $\mathrm{K}_{\mathrm{T}}$ ): Das Verteilungsvolumen ist beim adipösen Patienten deutlich erhöht. Der Arzneistoff verteilt sich ins Fettgewebe (Tiefes Kompartiment $\mathrm{K}_{\mathrm{T}}$ ), das beim adipösen Patienten deutlich größer ist und somit auch über eine größere Aufnahmefähigkeit für das Pharmakon verfügt. (modifiziert nach Hanley et al. 2010)

Zur Berechnung einer körpergewichtsbasierten Initialdosis für adipöse Patienten dient bei lipophilen Arzneistoffen mit großem Verteilungsvolumen das Gesamtkörpergewicht (total body weight, TBW) als Anhaltspunkt.

Zur Berechnung einer körpergewichtsbasierten Initialdosis für adipöse Patienten dient bei lipophoben Arzneistoffen mit niedrigem Verteilungsvolumen das fettfreie Körpergewicht (lean body weight, LBW) als Anhaltspunkt.

Allerdings muss beachtet werden, dass vereinzelt substanzspezifische Ausnahmen von dieser Regel existieren.

\subsection{Clearance bei Adipositas}

Da sich, wie bereits erwähnt, die Clearance von Arzneimitteln vereinfacht als Summe der hepatischen und der renalen Clearance beschreiben lässt, erscheint es zweckmäßig, den Einfluss von Adipositas auf diese beiden Ausscheidungswege zu betrachten.

\section{Hepatische Clearance bei Adipositas}

Falls keine zusätzlichen Leberfunktionsstörungen vorliegen, weisen adipöse Patienten gegenüber Normalgewichtigen eine leicht erhöhte hepatische Clearance auf. Emery et al. (2003) konnten bei adipösen Patienten einen Anstieg der Aktivität der Cytochrom P450 Isoform 2E1 (CYP2E1) nachweisen, die nach Gewichtsabnahme wieder auf den Normalwert abfiel. Allerdings wird die Relevanz dieser Beobachtung für die Arzneimitteltherapie kritisch bewertet, da nur wenige Pharmaka Substrate von CYP2E1 sind. Abernethy et al. (1983) konnten zusätzlich nachweisen, dass auch bei den sogenannten Phase-II-Reaktionen (Glucuronidierung und Sulfatierung) Anstiege der Aktivität bei adipösen Patienten zu verzeichnen sind. Häufig tritt bei adipösen Patienten allerdings eine nichtalkoholische Fettleber-Erkrankung auf, die, je nach Schweregrad, die hepatische Stoffwechselleistung beeinträchtigen kann, was folglich wiederum zu einer Verringerung der hepatischen Clearance führt.

\section{Renale Clearance bei Adipositas}

Die renale Clearance ist bei adipösen Patienten ohne sonstige Nierenerkrankungen erhöht. Henegar et al. (2001) führen diese Beobachtung im Wesentlichen auf die bei adipösen Menschen erhöhte Nierenmasse sowie den bei Adipositas erhöhten renalen Blutfluss zurück. Diese verstärkte Nierendurchblutung geht mit der bei Adipösen beobachteten Erhöhung des Herzminutenvolumens einher. Da fettleibige Patienten allerdings häufig auch an Typ-2-Diabetes leiden, muss berücksichtig werden, dass die Nierenfunktion eventuell infolge einer diabetischen Nephropathie eingeschränkt sein kann.

\section{Gesamtkörperclearance bei Adipositas}

Aus den obigen Erläuterungen geht hervor, dass die Gesamkörperclearance bei adipösen Patienten ohne renale oder hepatische Begleiterkrankungen erhöht ist. Han et al. (2007) konnten nachweisen, dass diese Zunahme der Gesamtkörperclearance linear mit der Zunahme der fettfreien Körpermasse (LBW) korreliert. Diese Feststellung steht in Zusammenhang mit der Tatsache, dass 99\% der metabolischen Prozesse im Körper und damit auch die Clearance von Pharmaka außerhalb des Fettgewebes lokalisiert sind. 


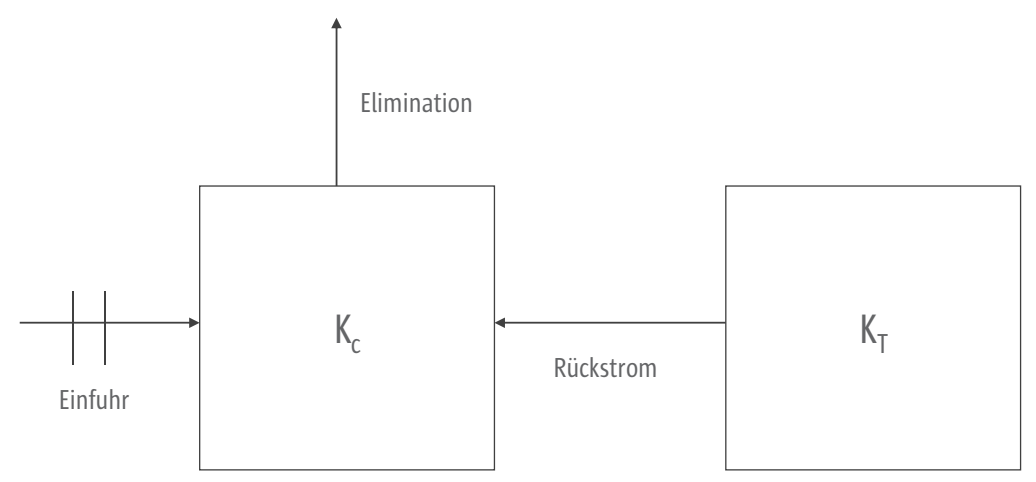

Abb. 7 Depoteffekt. Nach Absetzen einer lipophilen Medikation mit Tendenz zur Anreicherung im Fettgewebe (tiefes Kompartiment $\mathrm{K}_{\mathrm{T}}$ ) strömt noch längere Zeit die im Fettgewebe angereicherte Arzneistoffmenge ins zentrale Kompartiment zurück, wo schließlich die Elimination erfolgt. Durch den Rückstrom aus $\mathrm{K}_{\mathrm{T}}$ wird die Halbwertszeit verlängert. $\mathrm{K}_{\mathrm{T}}$ wirkt folglich wie ein Depot.

Da, wie in Kapitel VIII.1.2.2 beschrieben, die Clearance der zentrale pharmakokinetische Parameter zur Abschätzung der Erhaltungsdosis im steady state ist, sollte bei körpergewichtsbasierter Dosierung die fettreie Körpermasse zur Berechnung der Dosis verwendet werden. Bei Verwendung des Cesamtkörpergewichts besteht dagegen das Risiko einer zu hoch gewählten Erhaltungsdosis, was zu einer gefährlichen Kumulation des applizierten Wirkstoffs führen kann.

\section{Zur Berechnung einer körpergewichtsbasierten Erhal- tungsdosis dient bei adipösen Patienten die fettfreie Körpermasse als Anhaltspunkt.}

\section{Halbwertszeit bei Adipositas}

Da die Halbwertszeit als sekundärer pharmakokinetischer Parameter von den primären pharmakokinetischen Parametern Verteilungsvolumen und Clearance bestimmt wird, ist bei adipösen Patienten von einer Beeinflussung der Halbwertszeit infolge von verändertem Verteilungsvolumen und/oder Clearance auszugehen. Besonders lipophile Pharmaka weisen durch ihre Anreicherung im Fettgewebe bei adipösen Patienten häufig eine verlängerte Halbwertszeit auf, da bei Absetzen der Medikation das im Fettgewebe angereicherte Pharmakon langsam aus dem Fettgewebe zurück ins Zentralkompartiment strömt (Depoteffekt; s. Abb. 7). Der langsame Rückstrom aus dem Fettgewebe resultiert aus der dort physiologisch niedrigen Durchblutungsrate.

\section{Exkurs: Berechnung der fettfreien Körpermasse}

Zur Berechnung von Dosierungen ist in manchen Fällen die fettfreie Körpermasse (LBW) besser geeignet als das Gesamtkörpergewicht. Diese muss daher im klinischen Alltag schnell und unkompliziert berechnet werden können.

Janmasahatian et al. (2005) etablierten hierzu anhand von Daten aus der Bio-Impedanz-Analyse folgende Berechnungsmethode, die in der aktuellen Literatur als am besten geeignete Berechnungsweise bewertet wird. Hierbei wird aus dem Body-Mass-Index (BMI) sowie dem Gesamtgewicht die fettfreie Körpermasse errechnet (s. Abb. 8 u. 9).

Alternativ wird in der Literatur auch gelegentlich das ideale Körpergewicht (IBW) zur Berechnung adaptierter Dosierungen bei Adipositas verwendet. Diese Methode wird aber nach derzeitigem Kenntnisstand als weniger geeignet bewertet als die Berechnung mittels LBW, da bei der Berechnung des IBW lediglich Größe und Geschlecht berücksichtigt werden. So erhalten alle Patienten gleicher Größe und gleichen Geschlechts dieselbe Dosierung, ohne dass das Ausmaß der Adipositas mitberücksichtigt wird. Das ideale Körpergewicht wird nach folgenden Formeln berechnet.

Weibliche Patienten: IBW [in kg] = 45,4 + 0,89 x (Körpergröße in $\mathrm{cm}$ - 152,4)

Männliche Patienten: IBW [in kg] = 49,9 + 0,89 x (Körpergröße in $\mathrm{cm}$ - 152,4)

$L B W=\frac{9270 \times \text { TBW }}{8780+244 \times \text { BMI }}$

Abb. 8 Berechnung der fettfreien Körpermasse bei weiblichen Patienten 
$\mathrm{LBW}=\frac{9270 \times \mathrm{TBW}}{6680+216 \times \mathrm{BMI}}$

Abb. 9 Berechnung der fettfreien Körpermasse bei männlichen Patienten

\section{Abschätzung der Creatinin-Clearance bei adipösen Patienten}

Bei vielen Pharmaka wird in der Fachinformation eine Anpassung der Dosierung an die Nierenfunktion des Patienten empfohlen. In den meisten Fällen wird hierzu als Surrogat-Parameter für die glomeruläre Filtrationsrate die Creatinin-Clearance $(\mathrm{CrCl})$ mit Hilfe der Cockroft-Gault-Formel berechnet (s. Abb. 10).

Wenn allerdings bei adipösen Patienten das Gesamtkörpergewicht zur Berechnung der CreatininClearance genutzt wird, wird die GFR wesentlich zu hoch eingeschätzt, was folglich auch zu einer überhöhten Dosierung des Arzneimittels führt. Manjunath et al. (2010) empfehlen daher zur Berechnung der $\mathrm{CrCl}$ mittels der Cockroft-Gault-Formel bei adipösen Patienten an Stelle des Gesamtkörpergewichts

$\mathrm{CrCl}=\frac{(140-\text { Alter }) \times K G}{\mathrm{~S}_{\mathrm{cr}} \times 72}(\mathrm{x} 0,85 ;$ nur bei weiblichen Patienten $)$

Abb. 10 Cockroft-Gault-Formel zur Berechnung der CreatininClearance $\mathrm{CrCl}$ (in $\mathrm{ml} / \mathrm{min}$ ), $\mathrm{S}_{\text {cr }}$ : Serumkreatinin in $\mathrm{mg} / \mathrm{dl}, \mathrm{KG}$ : Körpergewicht in kg, Alter: Lebensalter in Jahren die fettfreie Körpermasse (LBW) zu verwenden. Dies erscheint sinnvoll, da gezeigt werden konnte, dass bei adipösen Patienten die GFR proportional zur Erhöhung der fettfreien Masse ansteigt.

Zur Berechnung der Creatinin-Clearance mit Hilfe der Cockroft-Gault-Formel sollte die fettfreie Körpermasse verwendet werden.

\section{Literatur}

Abernethy DR, Greenblatt DI, Divoll M, Shader RL (1983) Enhanced glucuronide conjugation of drugs in obesity: studies of lorazepam, oxazepam, and acetaminophen. I Lab Clin Med 101, $873-880$

Emery MG, Fisher JM, Chien JY, Kharasch ED, Dellinger EP, Kowdley KV, Thummel KE (2003) CYP2E1 activity before and after weight loss in morbidly obese subjects with non-alcoholic fatty liver disease. Hepatology 38, 428-35

Han PY, Duffull SB, Kirkpatrick CM, Green, B (2007) Dosing in obesity: a simple solution to a big problem. Clin Pharmacol Ther 82, 505-8

Hanley M), Darrell RA, Greenblatt D) (2010) Effect of obesity on the pharmacokinetics of drugs in humans. Clin Pharmacokinet 49, 71-87

Henegar JR, Bigler SA, Henegar LK, Tyagi SC, Hall JE (2001) Function$\mathrm{al}$ and structural changes in the kidney in the early stages of obesity. I Am Soc Nephrol 12, 1211-7

Janmahasatian D, Duffull SB, Ash S, Ward LC, Byrne NM, Green B (2005) Quantification of lean body weight. Clin Pharmacokinet 44, 1051-65

Manjunath P (2010) Estimating the glomerular filtration rate in obese adult patients for drug dosing. Advances in chronic kidney disease $5,53-62$

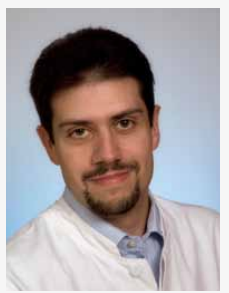

\section{Alexander Kratzer}

Studium der Pharmazie in Regensburg und Erhalt der Approbation 2008. Anschließend Tätigkeit als Klinischer Pharmazeut in der Apotheke der Krankenhäuser des Landkreises Landshut. Seit 2010 am Universitätsklinikum Regensburg tätig als Stationsapotheker auf internistischen und operativen Intensivstationen. Daneben klinisch-pharmazeutische Forschung auf dem Gebiet des Therapeutischen Drug-Monitoring in Kooperation mit dem Institut für Pharmazie Regensburg im Rahmen eines Promotionsprojekts. 